\title{
Germanica
}

\section{Gehirntiere und Schreckensmänner Intellektuelle Überlegenheit und Aggressivität im Frühwerk Arno Schmidts}

Gehirntiere et Schreckensmänner. Supériorité intellectuelle et agressivité dans l'œuvre d'Arno Schmidt des années 1940 et 1950

Gehirntiere and Schreckensmänner. Intellectual superiority and aggressiveness in the early work of Arno Schmidt.

\section{Jürgen Doll}

\section{OpenEdition}

\section{Journals}

Édition électronique

URL : http://journals.openedition.org/germanica/1385

DOI : 10.4000/germanica.1385

ISSN : 2107-0784

\section{Éditeur}

Université de Lille

Édition imprimée

Date de publication : 31 décembre 2011

Pagination : 121-133

ISBN : 9782913857285

ISSN : 0984-2632

\section{Référence électronique}

Jürgen Doll, « Gehirntiere und Schreckensmänner Intellektuelle Überlegenheit und Aggressivität im

Frühwerk Arno Schmidts », Germanica [Online], 49 | 2011, Online erschienen am: 26 März 2012, abgerufen am 06 Oktober 2020. URL : http://journals.openedition.org/germanica/1385 ; DOI : https:// doi.org/10.4000/germanica.1385

Ce document a été généré automatiquement le 6 octobre 2020.

(c) Tous droits réservés 


\section{Gehirntiere und Schreckensmänner Intellektuelle Überlegenheit und Aggressivität im Frühwerk Arno Schmidts}

Gehirntiere et Schreckensmänner. Supériorité intellectuelle et agressivité dans l'œuvre d'Arno Schmidt des années 1940 et 1950

Gehirntiere and Schreckensmänner. Intellectual superiority and aggressiveness in the early work of Arno Schmidt.

Jürgen Doll

Ein geistreicher Mensch hat, in gänzlicher Einsamkeit, an seinen eigenen Gedanken und Phantasien vortreffliche Unterhaltung, während von einem stumpfen die fortwährende Abwechslung von Gesellschaften, Schauspielen, Ausfahrten und Lustbarkeiten, die marternde Langeweile nicht abzuwehren vermag. ${ }^{1}$ Ich finde Niemanden, der so häufig recht hätte,

wie ich $!^{2}$

Dionysius von Halikarnassos stellt als Hauptforderungen an den Historiker : keine Religion; kein Vaterland; keine Freunde : das kannste haben ! ${ }^{3}$

1.

Als Arno Schmidt, in extremer finanzieller Notlage, ohne Verleger für seine Texte, zum ersten Mal Alfred Andersch, seinen unermüdlichen Förderer, aufsuchte, kam er 
keinesfalls als Bittsteller, sondern gab sich, wie die Protagonisten seiner Erzählungen, "geniebewusst, elitär und snobistisch“" ${ }^{4}$. Setzen sich die Alltagsgeschichten Schmidts in unterschiedlicher Gewichtung aus autobiographischen und fiktionalen Elementen zusammen, so reproduziert das Erzähler-Ich doch in hochgradigem Maße das Bewusstsein des Autors, dessen Lebensweg die Erzählungen - die antiken und utopischen ausgenommen - von der Angestelltentätigkeit in Schlesien über das Flüchtlingslager in Niedersachen bis zur Umsiedlung nach Rheinland-Pfalz nachzeichnen. Der 32jährige Protagonist von Brand's Haide (1951) heißt sogar Schmidt, ist Schriftsteller, wurde wie der gleichnamige Autor 1946 nach Cordingen verschlagen und schreibt wie dieser an einer Biographie Fouqués. Allerdings gewährt ihm der verheiratete Autor - um die in Schmidts Alltagsgeschichten obligate Liebesgeschichte zu ermöglichen - einen Junggesellenstatus und somit eine Freundin, die ihn jedoch, des (detailliert ausgemalten) Nachkriegselends müde, zugunsten eines reichen Vetters in Südamerika verlässt.

2 Schmidts Protagonisten sind (wie der Autor) durchwegs in sehr ärmlichen Verhältnissen lebende einzelgängerische, pessimistische, autodidaktische kleinbürgerliche Intellektuelle, auftretend mit der Pose des Genialen, interessiert an Mathematik und Naturwissenschaften, oft leidenschaftliche Sammler, die fasziniert sind von Statistiken, Tabellen, Messtischblättern, Realenzyklopädien. Sie pflegen archivarische Neigungen, studieren alte Akten, Landkarten, Staatshandbücher. Exzentrische Büchernarren - „denn es gibt keine Seligkeit ohne Bücher !“ $\left(\mathrm{E}^{5}, 120\right)$,verfügen sie über ein ausgedehntes, jederzeit bereitwillig ausgebreitetes faktisches Wissen, vorzüglich aus abgelegenen Gebieten („mein Fouqué : den möchte ich sehen, der davon nur halb so viel weiß wie ich !“ BH, 123). Unzählige literarische, historische, mythologische Anspielungen und Zitate, zuweilen als solche gekennzeichnet, aber meist in den Text eingearbeitet, zeugen vom enzyklopädischen Wissen der IchErzähler, bleiben dem Normalleser aber wohl häufig verborgen. ${ }^{6}$ So steht beispielsweise 83 Seiten Text von Brand's Haide ein Kommentar von 286 Seiten gegenüber. ${ }^{7}$ Das beständige name dropping, die Zitierwut, das Einfügen lateinischer oder fremdsprachlicher Ausdrücke und das Ausbreiten esoterischer Kenntnisse dienen den Helden der frühen Romane und Erzählungen vornehmlich dazu, ihren weltanschaulichen Gegnern oder auch den Frauen zu imponieren. So trumpft Düring vor dem Landrat mit seinen Kenntnissen der nichteuklidischen Geometrie auf (ALF, 32) und spielt Schmidt in Brand's Haide gezielt seine intellektuelle Überlegenheit vor Lore und Grete aus (BH, 103f.). Der Ich-Erzähler des utopischen Romans Schwarze Spiegel (1951) wiederum löst nach seiner Rückkehr aus dem zerstörten Hamburg „auf der Holztreppe vor dem Hause sitzend, das Fermatsche Problem“, wie Hans-Ulrich Treichel amüsiert anmerkt. ${ }^{8}$ Schmidts Ich-Erzähler leben in der Literatur und aus der Literatur, schulen ihre Sprache an der Literatur, an Wieland, Jean Paul, den Romantikern, Expressionisten wie August Stramm, Albert Ehrenstein oder Alfred Döblin. „Wieland ist mein größtes formales Erlebnis neben August Stramm !" erinnert Düring den Leser im Faun an seine Sprachvorbilder ${ }^{9}$ (ALF, 53). Schmidt erweist sich bereits in den frühen Erzählungen als äußerst hellhöriger, sprachschöpferischer, sprachexperimenteller Neuerer der deutschen Prosa, „der als genialisch hinhorchender, wenn auch aufsässiger Registrator mit katzenbergerischer Sammelwut die neue Zeit in ihrer neuen Sprache einfängt ${ }^{{ }_{10}}$. Die präzise Sprache, die originellen Bilder, die sprachlichen Neubildungen, der betont schnoddrige Ton wie der allgegenwärtige, meist bissige Humor und ironische Impetus erklären wohl neben dem freien, witzigen, oft betont 
vulgären Umgang mit erotischen Sujets ${ }^{11}$ und der politischen Aufsässigkeit der Protagonisten die Entstehung einer bedingungslosen Arno-Schmidt-Fangemeinde $a b$ den fünfziger Jahren.

Die Aufklärung bildet den Fluchtpunkt der Weltanschauung der Ich-Erzähler, die sich alle als Aufklärer verstehen. Allerdings bezieht sich Schmidt (und mit ihm seine IchErzähler) vorzüglich auf Spätaufklärer wie Wieland, Wezel oder Moritz, die bereits das Scheitern der Aufklärung an der Natur der Menschen thematisiert haben. Dennoch stellt ihr Pessimismus, der von Schmidts Protagonisten geteilt wird, die Einsichten der Aufklärung, den Glauben an die Vernunft nicht in Frage, sondern meldet Zweifel an deren Realisierbarkeit an. ${ }^{12}$ Es sind "die anderen“, die dem Ideal einer vernünftig organisierten Welt zuwider handeln, die Politiker, Militärs, Juristen, Lehrer, Journalisten, und auch „das Volk“, die stumpfsinnige, kulturlose Masse, „öde Gesichter, rübiges Gemüt, Gedankensteppe, Seelentundra“ (U, 101). Auf das Publikum im Landratsamt bezogen - um ein Beispiel eines in den Text integrierten Zitats anzuführen - wandelt der bildungsbewusste Düring einen Vers aus dem Lied von der Glocke ab : „Und der Kundschaft breitgestirnte glatte Scharen“" ${ }^{13}$ (ALF, 9). Schmidts Attacken gegen das „Volk“ rühren in erster Linie von seiner Enttäuschung über eine Bevölkerung her, die Hitler an die Macht gebracht und später den verhassten Adenauer unterstützt hat. So heißt es in Das steinerne Herz: „Dr. Adenauer (dem die Deutschen in einem ihrer periodisch $=$ üblichen - und nur dem Nichtkenner befremdlichen - Akte politischer Selbstentmannung die absolute Mehrheit gaben)“"14 (SH, 118-119). Einen Sonderfall bilden die als besonders rückständig und bigott angesehenen Bauern, denen die hinterhältigsten Attacken gelten: „40=Stunden=Woche'?: Nee! Die Landbevölkerung nicht reif : die würden lediglich 8 Stunden länger in der Kirche sitzen“ (SH, 165). Als Lösung sehen Schmidts Ich-Erzähler, die bundesdeutschen Leser provozierend, die Kollektivierung der Landwirtschaft wie in der DDR, die Bildung von Kolchosen vor.

Die Anknüpfung an die Aufklärung fundiert auch die radikale Kritik am Christentum und den militanten Atheismus der Schmidtschen Gehirntiere ${ }^{15}$ : „Ich?: Atheist, allerdings!: Wie jeder anständige Mensch!“ $(P, 45)$. Die verschiedenen in den Erzählungen auftretenden Pfarrer werden als besonders dumme, aufdringliche, dennoch ausgesprochen selbstgerechte Exemplare der Spezies Mensch vorgeführt. Sie wagen es zum Beispiel, selbst im Kriegsinferno von Leviathan (1949), die Sterbenden mit Berufung auf das Jenseits zu trösten. Doch spitzt sich Schmidts Polemik über die Kritik am „Machtapparat Kirche“ (L, 74), an den „Herren vom christlichen Gewerbe“ (SH, 38) hinaus auf eine prinzipielle Gegnerschaft gegen das Christentum zu : „Die Bibel : iss für mich ' $n$ unordentliches Buch mit 50000 Textvarianten. [...] und natürlich ewig merkwürdig durch den Einfluß, den es dank geschickter skrupelloser Propaganda und vor allem durch gemeinsten äußerlichen Zwang, compelle intrare, gehabt hat. $<$ Der Herr>, ohne dessen Willen kein Sperling vom Dache fällt oder 10 Millionen im KZ vergast werden, das müßte schon ne merkwürdige Type sein - wenn's ihn jetzt gäbe !“ $(P, 10)$. „Siehe, es war Alles gut !“ zitiert zynisch der Ich-Erzähler in Schwarze Spiegel angesichts der Verwüstungen des Kriegs (SP, 192). In der in der Spätantike spielenden Erzählung Kosmas oder Vom Berge des Nordens (1955), die die Auseinandersetzung zwischen der wissenschaftlich-vernunftorientierten Weltanschauung der kulturell hochstehenden Antike und der irrationalen, primitiven des triumphierenden Christentums schildert, will Schmidt das Christentum bereits in seinen Anfängen, 
sozusagen an der Wurzel, desavouieren : „Christentum und Kultur ? : das ist wie Wasser und Feuer ! ${ }^{{ }^{16}}$ (K, 228).

5 Angesichts der Vernunftlosigkeit der Welt bleiben die Schmidtschen Alltagshelden, allein im Besitz der Vernunft, einsam inmitten einer feindlichen Umgebung. Die Tatsache, dass alles ausschließlich aus dem Bewusstsein des Erzähler-Ichs berichtet wird, dessen private Meinungen, Obsessionen, Idiosynkrasien und Vorurteile spiegelt, verstärkt dieses Gefühl der völligen Isolierung. Wie Arno Schmidt in sein Holzhäuschen in Bargfeld, ziehen sich die Erzähler zurück in eine Blockhütte im Wald wie Düring im Faun, träumen von einer solchen wie Schmidt in Brand's Haide. Selbst der Held von Schwarze Spiegel, der glaubt, als Einziger den Atomkrieg überlebt zu haben, richtet sich nicht in einem der stehen gebliebenen Häuser ein, sondern baut sich aus dem auf langen Wanderungen zusammen gesammelten Material eine Hütte. Diese drei später in Nobodaddy's Kinder (1963) vereinigten Erzählungen sind Robinsonaden, in denen die Hütte ein Asyl bildet wie die Insel Felsenburg in der von Schmidt so geschätzten Robinsonade Schnabels. Am radikalsten - wenn auch ironisch gebrochen - äußert sich die hochgradige Misanthropie der frühen Helden in der Endzeitgeschichte Schwarze Spiegel, wo der allein zurück gebliebene Mensch sich „auf die herrliche einsame Zeit, viele Jahre lang“ (SP, 193) freut und in dem keineswegs verzweifelten Ausruf „ich brauche Niemanden !“ (SP, 180) sich seiner Einzigartigkeit versichert. Die Geistestiere fliehen jegliche Gemeinschaft, Familie, Verein, Kirche, Staat, wie Düring, der zuerst feststellt, er sei eigentlich immer Einzelgänger gewesen, um sodann rundheraus $\mathrm{zu}$ erklären : „Und wo Menschen in Scharen auftreten, immer den Rücken gedreht !“ (ALF, 20, 46). Desgleichen flüchten sie in möglichst alte Akten und Bücher. Dieser Rückzug sowohl in die Einsamkeit wie in antiquarische Studien wurde im Allgemeinen zu Recht als Selbstschutz der Helden interpretiert. In diesem Sinne schreibt Georg Klein in seinem schönen Nachwort zur Neuausgabe von Das steinerne Herz, der Protagonist verlasse seine Zeit und seine Welt, wo es nur gehe, in Richtung „Königreich Hannover“, und seine hoch spezialisierte Bücherliebe bilde „sein historisches Schutzgebiet vor den Zumutungen der Gegenwart“ (SH, 258, 259). „Das steinerne Herz: nur durch die dünne Nabelschnur der Staatshandbücherreihe hing die Welt noch an mir!“ (SH, 99) kommentiert der Ich-Erzähler mit verzweifelter Selbstironie.

Dieses misanthropische Einsiedlertum erinnert an Schopenhauer, den Schmidt gelegentlich zitiert und der, neben der Aufklärung (im weltanschaulichen) und der Romantik (im künstlerischen Bereich) die dritte wesentliche Referenz darstellt. Schmidts Figuren teilen durchweg dessen Menschen- und Weltverachtung, Pessimismus und Elitismus, aber auch dessen Glauben an Kunst und Wissenschaft als Palliative für die leidgeprägte Existenz. In Leviathan, der erstpublizierten Erzählung Arno Schmidts, die das erste Buch der Nachkriegszeit war, das Helmut Heißenbüttel beeindruckt hatte ${ }^{17}$, scheint die Anlehnung an Schopenhauer am engsten. In dieser im Chaos der letzten Kriegstage spielenden Erzählung herrscht der böse Dämon Leviathan. Der Wahnsinn des Krieges gibt dem namenlosen Helden sozusagen Schopenhauersche Sentenzen ein : „Diese Welt ist etwas, das besser nicht wäre; wer anders sagt, lügt! Denken Sie an die Weltmechanismen : Fressen und Geilheit. Wuchern und Ersticken." $(\mathrm{E}, 69)$. In Schwarze Spiegel dominiert weiterhin der Traum vom Ende der Welt („Bloß gut, daß Alles zu Ende war; und ich spuckte aus: Ende!“, SP, 172), in den nächsten Büchern ist der Wunsch nach Vernichtung der Menschheit abgeschwächt zur Forderung nach radikaler Geburtenbeschränkung. Doch suchen und finden die Schmidtschen Geistesmenschen, wie bei Schopenhauer, in der intellektuellen und 
künstlerischen Tätigkeit, in der Phantasie und im Denken einen Zufluchtsort. Bereits in Enthymesis oder W. I. E. H., dessen Untertitel immerhin „Wie ich Euch hasse“ meint, heißt es: „Wer nur kann groß sein? Künstler und Wissenschaftler! Und sonst niemand! Und von ihnen ist der kleinste Ehrliche tausendmal größer als der große Xerxes.“ (E, 105). In Brand's Haide erklärt Schmidt seiner Freundin : „Kunst überhaupt ! [...] für mich ist das Atemluft, das einzig Nötige, und alles Andere Klo und Notdurft" (BH, 137), und der Faun resümiert, bevor er seine Blockhütte anzündet : ,'Am Ende'- ich tappte mit der Handfläche auf mich Beleg : ,bleibt nur : Kunstwerke ; Naturschönheit ; Reine Wissenschaften. In dieser heiligen Trinität”“ (ALF, 88).

7 So düster die ersten drei, in Leviathan gesammelten Erzählungen sind, so sehr ist der misanthropische Grundton in allen folgenden durch zuweilen anzügliche Sexszenen, Humor und Sprachwitz aufgelockert, ja die Ironie richtet sich, zumal in Das steinerne Herz, gegen die Schwarzsicht, die gnadenlose Besserwisserei, den Rückzug des Helden in antiquarische Studien, ein prononciert selbstironischer Zug, der noch nicht genügend erkannt worden ist. ${ }^{18}$ Der Ich-Erzähler von Das steinerne Herz, der in schäbiger Berechnung ein Liebesverhältnis beginnt, nur um an die Staatshandbücher des Königreichs Hannover heranzukommen, der mit einer perfekt ausgeklügelten Strategie einen alten Folianten aus der Bibliothek entwendet und nach der Entdeckung eines Goldschatzes beschließt, bei der Geliebten zu bleiben, die er grußlos verlassen wollte, ist nicht gerade das Muster eines integren Intellektuellen. Und er scheint es selbst zu wissen, wenn man sich an seine zahlreichen selbstironischen Beobachtungen hält. Als er sich ohne Abschied von seiner Geliebten davonmachen will, rechtfertigt er sich wie folgt : „wir Superklugen legten nicht solchen Wert auf menschliche Verhältnisse“ (SH, 158). Auch seine geliebten Staatshandbücher fallen dem Spott zum Opfer : „Ich kann keinen Menschen achten, der nicht hannoversche Staatshandbücher sammelt !“ (SH, 215f.). Vollends überzeugt von der Neigung des Helden zur Selbstironie sind wir, wenn wir auf folgenden, in absichtlich ungewöhnlich pathetischer Sprache formulierten Gedanken treffen: „Und kein Handexemplar! Tiefe Niedergeschlagenheit überkam mich: nie würde ich den Druckfehler auf Seite 260 wissen!“, SH, 198). Im recht spöttischen utopischen Roman Die Gelehrtenrepublik (1957), dem letzten Titel seines Frühwerks, macht sich Schmidt über seine pedantische Faktenhuberei in den witzigen Fußnoten des Übersetzers Chr. M. Stadion (Anagramm aus Arno Schmidt) lustig. ${ }^{19}$

\section{2.}

8 Schmidts frühe Erzählungen und Romane verstehen sich auch als detailgesättigte Alltagsprotokolle der Nazizeit und Nachkriegsdeutschlands. Aus dem Leben eines Fauns spielt in einer schlesischen Kleinstadt in den Jahren 1939 und 1944, Leviathan im Februar 1945, Brand's Haide in einem Flüchtlingslager 1946, Die Umsiedler wie die Urlaubsromanze Seelandschaft mit Pocahontas Anfang der 1950er Jahre, Das steinerne Herz im Jahre 1954. Bei der Kommentierung der politischen, sozialen, kulturellen Verhältnisse jener Jahre erweisen sich die Protagonisten als Erben der plebejischen Schreckensmänner aus Schmidts Funkfeatures. „Intelligenz lähmt, schwächt, hindert ? Ihr werd't Euch wundern!: Scharf wie 'n Terrier macht se!!“, erklärt Eggers programmatisch gleich zu Beginn von Das steinerne Herz (SH, 7). seiner schreibenden Zeitgenossen. So werden in Leviathan der Nationalsozialismus und 
der Krieg dämonisiert, wird die Eroberungspolitik der Nazis in der 1948 entstandenen „antiken“ Erzählung Alexander oder was ist Wahrheit, einer durchsichtigen Darstellung Nazi-Deutschlands mit Lebensborn, Herrenrasse, Horst-Wessel-Lied und offiziersverschwörung, auf den Größenwahn und schließlich den Wahnsinn AlexanderHitlers zurückgeführt.

Im Kurzroman Aus dem Leben eines Fauns, der den Alltag in einem Landratsamt unter dem Nationalsozialismus schildert, dominiert, wohl Schmidts eigenem Erleben gemäß, „die doppelte Buchführung des Lebens“ (Wolfram Schütte ${ }^{20}$ ), die Kluft zwischen äußerer Anpassung und innerer Überzeugung. Die tiefempfundene Gegnerschaft des Helden zum Regime spielt sich wider Willen ausschließlich in seinem Kopf ab : „Nur schade, daß ich, ein Sehender, das Blinde-Kuh-Spiel werde mitmachen müssen. (Na, vielleicht kann man doch etwas beiseite treten. Ma sehn).“ (ALF, 15). In der Tat hält Düring sich bedeckt : „<HeilittlerSiewünschen ?> [...] Also hob ich [die Rechte] leicht zum Deutschen Gruß, und ballte dafür die freie Linke“, ALF, 26) und tröstet sich mit seiner geistigen Opposition : „Hab [...] two separate sides to my head, während die Braunen nur eine haben." (ALF, 56).

11 Seine prinzipielle Ablehnung jeglicher Gemeinschaft, von Staat, Parteien, Organisationen, seine individualanarchistische Verdammung jeglicher Autorität erlaubt ihm nicht immer, die Besonderheit des Nazi-Regimes richtig einzuschätzen. Er ist nicht der Sehende, der er zu sein glaubt. Als Runge, einer seiner Bürokollegen, vom Dienst als SS-Mann in Bergen-Belsen zurückkommt und stolz von den Spezialgalgen berichtet $^{21}$, an denen die Juden aufgehängt werden, reagiert Düring mit einer allgemeinen Politikerbeschimpfung: „Nichts! Ich weiß nichts! Ich kümmre mich um nichts! (Aber das weiß ich : Alle Politiker, alle Generäle, alle irgendwie Herrschenden oder Befehlenden sind Schufte! Ohne Ausnahme! Alle !“ (ALF, 14). Ähnlich fragwürdig scheint es, wenn der Held von Leviathan, von seinem antiklerikalen Furor verleitet, die KZs in die Schreckensgeschichte der Kirche einfügt : „Denn erfunden ist ja nicht von Stalin oder Hitler oder im Burenkrieg das Konzentrationslager, sondern im Schoße der heiligen Inquisition; und die erste abendländisch exakte Schilderung eines wohleingerichteten K. Z. verdanken wir ja der allerchristlichst pervertierten Phantasie Dantes - bitte, es fehlt nichts: die Jauchengruben, die Eiswasserfolter, der ewige Laufschritt der klatschend Geprügelten; [...] Écrasez l'infâme!" (L, 74). Die hier zitierten Stellen gehören zu den wenigen, in denen von der Judenverfolgung die Rede ist, und die geringe Bedeutung dieses Themas wurde Schmidt häufig vorgeworfen. Doch unterscheidet er sich damit in keiner Weise von seinen schriftstellernden Zeitgenossen, was auch Jörg Drews, der wohl beste Kenner und kompetenteste Fürsprecher seines Werks, eingeräumt hat. „Die Literatur bis zum Ende der fünfziger Jahre“ schreibt Drews, „war eine Literatur von Opfern. Die Art von Schuld, die man, auch wenn man nicht selbst gemordet hat, als Deutscher auf sich geladen hatte, wurde höchstens summarisch anerkannt; es wurde ihr aber nicht im Detail nachgegangen, übrigens auch nicht bei Borchert, Koeppen, Böll oder Andersch, (in gewissem Sinn ja auch nicht bei Arno Schmidt, aber das wäre ein besonderes Kapitel)““22.

Auch Schmidts Nachkriegserzählungen waren in der Tat eine Literatur der Opfer. Dabei handelt es sich weniger um die Landser, wie etwa bei Böll, wenn auch deprimierende Kriegserlebnisse, vor allem in Form von Schikanen der Offiziere, in den Gesprächen immer wieder auftauchen, als um Heimkehrer (wie bei seinen Zeitgenossen), Flüchtlinge, Heimatvertriebene, Umsiedler. Doch sind diese Opfer frei von allem 
Selbstmitleid und zeichnen sich als scharfzüngige, aggressive Kritiker der bestehenden Verhältnisse aus. Schon Heißenbüttel wies am Beispiel des Helden von Brand's Haide darauf hin, dass sie ihre stärkste Aktivität in der Kritik entwickelt hätten. ${ }^{23}$ Sie lassen kein gutes Haar an Politik, Militär, Polizei, Kirche, dem sich auf Illustrierte, Schlager und Fritz-Walter-Kult beschränkenden Kulturbetrieb, schimpfen zügellos auf Adenauer, die „Firma Pacelli \& Eisenhower“ (SH, 119) und das leicht zu verführende Volk. Die Invektiven beschränken sich nun nicht mehr nur auf Assoziationen des Protagonisten, sondern werden im Gespräch geäußert, wobei seine Gesprächspartner, wie Erich in Seelandschaft mit Pocahontas oder Karl Thumann in Das steinerne Herz, beide SPD-Anhänger, im Wesentlichen seine Ansichten teilen. Auffällig ist seine Demokratiekritik, die wiederholte Forderung nach einer Prüfung, der sich die Wahlberechtigten unterziehen müssten, wie beispielsweise in Seelandschaft mit Pocahontas: „(Aber mal ernsthaft: allgemeines und gleiches Wahlrecht ist Unsinn: zumindest müßte Jeder erst 'ne geschichtlich-geographische Prüfung ablegen ; und mit 65 Jahren das Wahlrecht, aktiv wie passiv, überhaupt erlöschen!)“ (SH, 22). Es ist nicht klar zu entscheiden, ob diese Forderung dem Elitismus des Protagonisten geschuldet ist oder seiner Verärgerung darüber, dass die Mehrheit CDU und Adenauer wählt (,aber dafür Wiederaufrüstung he : was müssen das für Ochsen sein, die sich den Fleischer zum König wählen !“, P, 74).

13 Adenauer ist die Hauptzielscheibe der Angriffe der Schmidtschen Helden. AdenauerDeutschland ist ihnen derart zuwider, dass etwa in Das steinerne Herz, einem der ersten Romane, der vom geteilten Deutschland handelt, die Bundesrepublik wie die DDR gleichermaßen und eigentlich in gleichem Maße kritisiert werden, ja die DDR manchmal besser wegzukommen scheint. Der Hauptvorwurf an Adenauer ist seine Politik der Wiederaufrüstung. Jede andere Kritik, wie die an Adenauers Westorientierung, seiner die Wiedervereinigung blockierenden Politik („Iss doch ganz klaa : entweder EVG oder Wiedervereinigung; Beedes gipts nich !“, P, 33) oder an der Beschäftigung ehemaliger Nazis, ist der an der Wiederaufrüstung untergeordnet. So lesen wir über die rehabilitierten Nazis in Das steinerne Herz: „Gewiß : ein Regime, das aufrüsten will, muß doch einfach auf jene zurückgreifen, die damals bei Hitler oben schwammen“ (SH, 90). Die Wiederaufrüstung macht für Schmidt die Nachkriegsjahre zu einer neuen Vorkriegszeit. Davon zeugen die utopischen Romane, die nach einem atomaren Krieg spielen. In Schwarze Spiegel (1951) findet dieser bereits 1955 statt, in Die Gelehrtenrepublik (1957) und in dem späteren Kaff, auch Mare Crisium (1960) in den 1970er Jahren. Der „Rüstungsalp“ (SH, 153) Adenauer wird in diesen frühen Erzählungen als bösartiger seniler Politiker dargestellt: „aber der Staatsmann, Senilissimus, wird scheinbar erst mit 75 so recht reif, eiskalt, total unmenschlich, greisig gräulich griesgram Gräber grimmig." ( $P, 74)$. Selbst die enge Anbindung Adenauers an die katholische Kirche, die den atheistischen Helden Schmidts ein Abscheu ist, wird im Zusammenhang mit der Rüstungspolitik gesehen: so meint Eggers, die Ostzone interessiere Adenauer nicht, denn, stimmte der evangelische Osten mit, würde „sein aus Kanonen und Kruzifixen gebastelter Thron [zusammenstürzen].“ (SH, 119). Insgesamt wird die Adenauersche Politik beinahe als Restauration der Nazizeit empfunden, was Karl Thumann der Weimarer Zeit als den „intelligentesten und freiesten, glaubens- und uniformlosesten Jahren“" nachtrauern lässt (SH, 13f.). Ja, die oppositionell eingestellten Figuren dieser Erzählungen fühlen sich sogar wieder bedroht. „,Bloß pollietisch mußte im Augenblick ganz vorsichtich sein'“, bemerkt Erich in Seelandschaft mit Pocahontas, , , na, ich geb Jed'm recht : und wähln tu ich doch was ich 
will !' (und vertraulich-neugierig, ganz wie früher, im Flüsterton des Dritten Reichs).“ $(\mathrm{P}, 14)$.

14 Die politischen Polemiken Schmidts und seiner Schreckensmänner, „Franks-tireurs des Geistes“ (U, 120), wurden in jüngerer Zeit immer wieder kritisiert. So vertrat beispielsweise Erich Fried 1978 die damals verbreitete Ansicht, Arno Schmidt sei „politisch vielleicht nur ein einigermaßen progressiver Stammtischpolitiker oder so etwas" ${ }^{\text {“24 }}$, und Schmidts Biograph Wolfgang Martynkewicz fordert den Leser auf, nicht zu übersehen, „dass die wirklichen Ereignisse, die historischen Katastrophen sich eher im Hintergrund abspielen und nur als Zitate vorkommen “25. Erich Fried erwartete in seiner hochpolitischen Periode wohl, Schmidts Ich-Erzähler sollten eine zusammenhängende (linke) politische Analyse anbieten und sich womöglich engagieren oder gar organisieren. Wie aber sollte das möglich sein bei so notorischen Einzelgängern, denen jedes gemeinschaftliche Handeln zutiefst zuwider ist, die, wie der Protagonist von Die Umsiedler, das Gefühl haben, geistig befänden sie sich lebenslänglich in Einzelhaft $(U, 74)$ ? An eine Erzählung oder einen Roman die Forderung zu stellen, die Geschichte müsse deren Vordergrund abgeben, wie es Martynkewicz zu tun scheint, scheint mir geradezu abwegig. Ebenso wenig scheint mir seine Definition von Gedanken- und Gesprächssplittern als Zitaten Schmidts Technik der Momentaufnahme, des Schnappschusses, gerecht zu werden. Beide Kritiker scheinen zu übersehen, dass in Schmidts Alltagserzählungen Zeitgeschichte und Politik, ausschließlich aus der Perspektive der Protagonisten vergegenwärtigt wird. Beide verkennen so die Absicht des Autors, Geschichte, Politik, Religion, Kultur eben nicht argumentativ abzuhandeln, sondern in der Assoziationstätigkeit der Figuren und in Alltagsgesprächen aufblitzen und wieder verschwinden zu lassen. Auch mag man die apodiktischen Urteile und Schimpftiraden der Helden, die durch den Hass auf die Wiederaufrüstung und die Angst vor einem neuen Krieg motiviert sind, die Nase rümpfend dem Stammtisch zuordnen. Aber erwartet man wirklich, in Alltagsgeschichten aus der NS-Vergangenheit und der Nachkriegs-Gegenwart Figuren ohne Vorurteile, Vorlieben, Ticks und Ressentiments zu finden?

Vor allem scheinen die nach dem außerordentlichen Liberalisierungsschub in den 1970er Jahren, Ende der 1970er und Anfang der 1980er Jahre gefällten Urteile den geschichtlichen Kontext, in dem Schmidts Frühwerk entstanden ist, zu vernachlässigen. Vergessen wir nicht, dass 1955 gegen Seelandschaft mit Pocahontas Anklage wegen Gotteslästerung und Verbreitung unzüchtiger Schriften erhoben wurde, was Schmidt veranlasste, aus dem schwarzen Rheinland-Pfalz nach SPD-Hessen umzuziehen, und dass Das steinerne Herz 1956 nur in einer politisch purgierten Fassung veröffentlicht werden konnte. (Der Urtext erschien erst 1986.) Damals fanden die politischen Schimpfereien, auch wenn sie in gedanklichen Assoziationen und Alltagsgesprächen verpackt waren, verständlicherweise durchaus die Gunst politisch renitenter Leser. Und auch heute noch werden (kritische) Leser, verführt von Schmidts Sprachkunst und erzählerischem Erfindungsgeist, scharf ironische politische Passagen wie folgende als immer noch aktuell empfinden können: „'Was ist demnach das beste Rezept für ein Erdenleben überhaupt, oben wie unten?',Aufs Dorf ziehen. Doof sein. Rammeln. Maul halten. Kirche gehen. Wenn $\mathrm{n}$ großer Mann in der Nähe auftaucht, in $\mathrm{n}$ Stall verschwinden : dahin kommt er kaum nach! Gegen Schreib = und Leseunterricht stimmen ; für die Wiederaufrüstung! Atombomben !'“26. 


\section{Siglen und verwendete Ausgaben}

A Alexander oder was ist die Wahrheit [1953], in : Rosen \& Porree, Frankfurt am Main, Fischer Verlag, 1984, S. 125-179.

ALF Aus dem Leben eines Fauns [1953], in : Nobodaddy's Kinder, Reinbek bei Hamburg, Rowohlt, 1963, S. 5-90.

BH Brand's Haide [1951], in : Nobodaddy's Kinder, Reinbek bei Hamburg, Rowohlt, 1963, S. 91-168.

E Enthymesis oder W. I. E. H. [1949], in : Leviathan, Frankfurt am Main, Suhrkamp, 1963, S. 83-127 (Bibliothek Suhrkamp 104).

G Die Gelehrtenrepublik [1957], Frankfurt am Main, Fischer Verlag, 1965 (Fischer Bücherei 685).

K Kosmas oder Vom Berg des Nordens [1955], in : Rosen \& Porree, Frankfurt am Main, Fischer Verlag, 1984, S. 183-279.

L Leviathan [1949], in : Leviathan, Frankfurt am Main, Suhrkamp, 1963, S. $43-81$ (Bibliothek Suhrkamp 104).

P Seelandschaft mit Pocahontas [1955], in : Rosen \& Porree, Frankfurt am Main, Fischer Verlag, 1984, S. 7-69.

SH Das steinerne Herz. Historischer Roman aus dem Jahre 1954 nach Christi [1956], Frankfurt am Main, Suhrkamp, 2002 (Bibliothek Suhrkamp 1353).

SP Schwarze Spiegel [1951], in : Nobodaddy's Kinder, Reinbek bei Hamburg, Rowohlt, 1963, S. 169-226.

U Die Umsiedler [1953], in : Rosen \& Porree, Frankfurt am Main, Fischer Verlag, 1984, S. 73-121.

\section{NOTES}

1. Arthur Schopenhauer, Aphorismen zur Lebensweisheit, Frankfurt am Main, Insel-Verlag, 1980, S. 15.

2. A. Schmidt, Die Umsiedler, in : Rosen \& Porree, Frankfurt am Main, Fischer, 1984, S. 71-121, hier S. 90.

3. A. Schmidt, Das steinerne Herz, Frankfurt am Main, Suhrkamp, 2002, S. 79.

4. Stephan Reinhardt, Alfred Andersch, Zürich, Diogenes, 1996, S. 202, zit. in.: Wolfgang Martynkewicz, Arno Schmidt, Reinbek bei Hamburg, Rowohlt, 1992, S. 66.

5. Ein Siglenverzeichnis ist am Ende des Aufsatzes aufgeführt.

6. Selbst wenn der Stellenwert des oft aus zweiter Hand stammenden Bildungszitats inzwischen als prekär eingeschätzt wird, bleibt dessen Funktion im Textgefüge unverändert (vgl. etwa HansUlrich Treichel, „Bilder vom letzten Menschen. Anmerkungen zum Frühwerk Arno Schmidts“, in : H.-U. T., Über die Schrift hinaus. Essays zur Literatur, Frankfurt am Main, Suhrkamp, 2000, S. 150-168, hier S. 159f.)

7. Heinrich Schwier, Lore, Grete \& Schmidt. Ein kommentierendes Handbuch zu Arno Schmidts Roman „Brand's Haide“, München, edition text+kritik, 2000. Bekanntlich bildete sich in den 
sechziger Jahren ein Arno-Schmidt-Dechiffrierungssyndikat mit dem Ziel, alle Anspielungen und Zitate aufzufinden und zu erklären.

8. H.-U. Treichel, op. cit., S. 154.

9. Vgl. auch : „Einmal neigte ich den Kopf, das Haupt, vor August Stramm : dem großen Dichter ! (Auch Albert Ehrenstein, sagt, was ihr wollt!)“ (SP, 178).

10. Robert Minder, „Lüneburger Heide, Worpswede und andere Heide- und Moorlandschaften“, in : R. M., Acht Essays zur Literatur, Frankfurt, Fischer 1969, S. 59-75, hier S. 75.

11. Ein Beispiel unter vielen: „In Ihrer Haut möcht ich stecken, Fräulein Krämer!“ (Schönert, beklommen seufzend. Noch mal) : „In Ihrer Haut möcht ich stecken.“ Sie sah ihn mißtrauisch aus den langen Augenwinkeln an (hat ja wohl auch ihre Sorgen). - „Dochdoch“, beteuerte er fromm, „Und wenns son Stück wär : - zeigte : etwa 20 Zentimeter. - Ihr Mund, zuerst verblüfft plissiert, löste sich auf, in eddies and dimples, dann prustete sie semig (selbst ich griente würdig und abteilungsleitern : der Schönert, das Schwein. Ja, der war unverheiratet !)“ (ALF, 9).

12. Siehe dazu Horst Thomé, Natur und Geschichte im Frühwerk Arno Schmidts, München, edition text + kritik, 1981, S. 47-92.

13. In „Das Lied von der Glocke“ heißt es bekanntlich : „Und der Rinder/ Breitgestirnte, glatte Scharen/ Kommen brüllend,/ Die gewohnten Ställe füllend“.

14. Dieser Satz konnte in der purgierten Erstausgabe nicht publiziert werden.

15. Die Begriffe "Gehirntiere" und "Schreckensmänner" hat Schmidt in seinen literarhistorischen Funkessays, etwa in „Müller oder vom Gehirntier“ und „Die Schreckensmänner. Karl Philipp Moritz zum 200. Geburtstag“ entwickelt. Die Schreckensmänner gehören ihrem sozialen Status nach der plebejisch-kleinbürgerlichen literarischen Intelligenz an und verbinden überlegene Intellektualität mit politischem Rebellionsgeist.

16. Von seiner antichristlichen Obsession angetrieben scheut sich Schmidt in Kosmas nicht, die christliche Wissenschaft an die „deutsche Physik“ der Nazis anzunähern: „<ChristianikeTopographia> ? ! allein der Titel ist ja ein Ding für sich : ungefähr, als wenn ich <Thrakische Mathematik> schreiben wollte!“ (K, 195f.). In Leviathan schließlich scheint er die Pfarrer der SS gleichzustellen: „blinde Gefolgschaft scheint immer schwarze Uniformen zu tragen“ $(\mathrm{L}, 61)$.

17. H. Heißenbüttel, „1945 ist heute. Ein persönlicher Bericht“, in: Literaturmagazin 7 : Nachkriegsliteratur, hrsg. von Nicolas Born u. Jürgen Manthey, Reinbek, Rowohlt, 1977, S. 232-236, hier S. 235.

18. Helmut Heißenbüttel etwa schreibt in seinem schönen Aufsatz über Schmidt, erst in Kaff, auch Mare Crisium (1960), erscheine der Erzähler zum ersten Mal ironisch gebrochen (H. H., „Annäherung an Arno Schmidt", in H. H., Über Literatur, München, sonderreihe dtv, 1966, S. 51-65, hier S. 64.)

19. Durchgehend selbstironisch ist die Erzählperspektive in den beiden vergnüglichen Erzählungen Tina oder über die Unsterblichkeit (1955) und Goethe und Einer seiner Bewunderer (1956), auf die hier nicht näher eingegangen werden kann. Vgl. z. B. aus Tina: „Ich fuhr herum: Wildenhayn? ?: Das wußte Niemand außer mir!! Wollte mich wieder ein Hund um die Priorität bringen?!“ (A. Schmidt, Tina. Goethe. Aus der Inselstraße. Stürenburg-Geschichten, Zürich, Haffmanns Verlag, 1985, S. 11). Aus Goethe : „Schmidt ? ? - - : ach so' sagte er [Goethe] gnädig, als ich stumm auf mich wies, und schmunzelte verständnisvoll : 'Gut. - - : sogar sehr gut !" (ebenda, S. 46).

20. W. Schütte, "Robinsonaden und Bibliomania oder : Kopf-Welten. Notizen zu den Anfängen Arno Schmidts“, in : Literaturmagazin 7 : Nachkriegsliteratur, hrsg. von Nicolas Born u. Jürgen Manthey, Reinbek, Rowohlt, 1977, S. 296-302, hier S. 298.

21. In Wirklichkeit existierte das Lager Bergen-Belsen 1939 noch nicht, es wurde erst 1943 eröffnet. Schmidt hätte also diese Episode im letzten Teil des Romans, der 1944 spielt, erzählen können, aber es ging ihm offenbar vor allem darum, dieses Thema prinzipiell einzubringen. 
22. J. Drews, „'Was ich nun sah, war über alle Beschreibunk!'. Arno Schmidts Kaff, auch Mare crisium und das Ende der ersten Phase der westdeutschen Nachkriegsliteratur", in: J. Drews/ Doris Plöschberger, Hrsg., Starker Toback, voller Glockenklang: zehn Studien zum Werk Arno Schmidts, Bielefeld, Aisthesis Verlag, 2001, S. 73-91, hier S. 79).

23. H. Heißenbüttel, „Annäherung an Arno Schmidt“, vgl. Anmerkung 18, S. 55.

24. E. Fried, „Lesen und Schreiben während des Exils. Interview mit Anke Winckler“, in : Lutz Winckler, Hrsg., Antifaschistische Literatur. Prosaformen, Bd. 3, Königstein/Ts., Scriptor Verlag, 1979, S. 269-284, hier S. $269 f$.

25. W. Martynkewicz, Arno Schmidt, Reinbek bei Hamburg, Rowohlt, 1992, S. 54.

26. A. Schmidt, Tina oder über die Unsterblichkeit (1955), in : Tina. Goethe. Aus der Inselstraße. Stürenburg-Geschichten, Zürich, Haffmanns Verlag, 1985, S. 29.

\section{RÉSUMÉS}

In einem ersten Teil wird versucht, das Profil der Ich-Erzähler in Schmidts frühen sprachexperimentellen Erzählungen und Romanen $\mathrm{zu}$ umreißen. Es handelt sich um einzelgängerische, geniebewusste, elitäre, eher linksorientierte kleinbürgerliche Intellektuelle, die ihre Umgebung durch Ausbreitung entlegener Kenntnisse und gnadenlose Besserwisserei zu beeindrucken suchen. Allerdings wird dieses eher abstoßende Charakterbild durch eine fast allgegenwärtige Ironie gebrochen, die sich gerade gegen die Ticks, Vorurteile, Idiosynkrasien und Ressentiments dieser Ich-Erzähler richtet. Im zweiten Teil soll gezeigt werden, inwiefern der überzogene Individualismus und Elitismus der Ich-Erzähler, ihre Demokratieskepsis und Ablehnung jeglicher Gemeinschaft ihre politische Beurteilung des Nazi-Regimes und AdenauerDeutschlands beeinflusst, wobei auch diesbezüglich Schmidts Hang zur Ironie und sein ausgeprägter Sinn für Provokation in Betracht gezogen werden müssen.

Dans la première partie de l'article, j'essaie d'esquisser le profil des narrateurs à la première personne des nouvelles et romans expérimentaux d'Arno Schmidt des années 1940 et 1950. Il s'agit d'intellectuels petit-bourgeois de gauche, solitaires, élitistes et imbus de leur supériorité qui veulent impressionner leur entourage par l'étalage de connaissances souvent ésotériques. Toutefois, ce profil plutôt repoussant est nuancé par une ironie presque omniprésente, qui vise précisément les manies, les préjugés, les idiosyncrasies et les ressentiments de ces narrateurs. Dans la seconde partie, je tente de montrer dans quelle mesure l'individualisme exacerbé et l'élitisme du narrateur, son scepticisme à l'égard de la démocratie et son refus de toute communauté ont pu peser sur son évaluation politique du régime nazi et de l'Allemagne d'Adenauer. Cependant, ici aussi, le regard ironique que Schmidt pose sur la réalité et son sens prononcé de la provocation relativisent les jugements tranchés qu'expriment les narrateurs.

In a first part we attempt to outline the profile of the narrators in Schmidt's early experimental stories and novels. They are solitary, elitist, left-leaning middle-class intellectuals who try to impress their fellow citizens by displaying vast and often esoteric knowledge. However, this rather repulsive portrait is softened by an almost ever-present irony, which is aimed precisely at the tics, prejudices, resentments and idiosyncrasies of the narrators. In the second part we try to show how the narrator's exacerbated individualism and elitism, his scepticism toward democracy and rejection of any community influenced his political assessment of the Nazi regime and 
Adenauer's Germany. In this respect too Schmidt's penchant for irony and his strong sense of provocation must be taken in consideration.

\section{AUTEUR}

\section{JÜRGEN DOLL}

Université Paris-Est Créteil Val-de-Marne (UPEC) 